\title{
Expression of $m i R-486$ is a potential prognostic factor after nephrectomy in advanced renal cell carcinoma
}

\author{
KEISUKE GOTO $^{1,2}$, NAOHIDE OUE ${ }^{1}$, SHUNSUKE SHINMEI ${ }^{1,2}$, KAZUHIRO SENTANI $^{1}$, NAOYA SAKAMOTO ${ }^{1}$, \\ YUTAKA NAITO $^{1}$, TETSUTARO HAYASHI ${ }^{2}$, JUN TEISHIMA ${ }^{2}$, AKIO MATSUBARA ${ }^{2}$ and WATARU YASUI ${ }^{1}$
}

Departments of ${ }^{1}$ Molecular Pathology and ${ }^{2}$ Urology, Hiroshima University Institute

of Biomedical and Health Sciences, Hiroshima 734-8551, Japan

Received October 3, 2012; Accepted November 6, 2012

DOI: $10.3892 / \mathrm{mco} .2012 .46$

\begin{abstract}
Renal cell carcinoma is the most frequently occurring neoplasm in the adult kidney, leading to poor prognosis. Therefore novel biomarkers are required for the prediction of early metastasis following nephrectomy. The aim of the present study was to investigate whether or not expression levels of $m i R-486$, detected in RNA isolated from formalin-fixed paraffin-embedded tissue sections, can predict prognosis for patients with renal cell carcinoma (RCC). Expression levels of $m i R-486$ were measured by quantitative reverse transcriptase-polymerase chain reaction in 150 RCC cases. Expression of $m i R-486$ in RCC samples was 2.7 -fold higher than in corresponding non-neoplastic kidney samples $(\mathrm{P}<0.0001)$. In stage III and IV RCC cases $(\mathrm{n}=46)$, a high $m i R-486$ expression in tumors was associated with worse cancer-specific mortality, independent of clinical covariates, including TNM staging ( $\mathrm{P}=0.0064)$. In addition, $m i R-486$ expression tended to be associated with cancer-specific mortality in stage III and IV RCC patients who were not treated with interferon- $\alpha$ (Kaplan-Meier analysis, $\mathrm{n}=14, \mathrm{P}=0.0574)$. These results suggest that $m i R-486$ is a promising biomarker to identify poor prognosis in RCC patients. As expression of $m i R-486$ was measured from formalin-fixed paraffin-embedded (FFPE) samples, this study demonstrated that measurement of $m i R-486$ may be readily translated into clinical applications.
\end{abstract}

\section{Introduction}

Renal cell carcinoma (RCC) is the most common neoplasm in the adult kidney and accounts for 2-3\% of malignant diseases in adults. Locally extensive or metastatic RCC, even following complete resection, have worse prognoses compared to

Correspondence to: Professor Wataru Yasui, Department of Molecular Pathology, Hiroshima University Institute of Biomedical and Health Sciences, 1-2-3 Kasumi, Minami-ku, Hiroshima 734-8551, Japan

E-mail: wyasui@hiroshima-u.ac.jp

Key words: miRNA, miR-486, renal cell carcinoma, prognosis organ-confined diseases. Therefore, there is a need to identify novel biomarkers that enable prediction of early metastasis after nephrectomy, and to develop novel targeted therapies.

Cancer develops as a result of multiple genetic and epigenetic alterations. Better knowledge of changes in gene expression that occur during carcinogenesis may lead to improvements in diagnosis, treatment and prevention. Potential biomarkers identifying high-risk patients have been reported, however, assessment methods for these biomarkers often involve RNA-based techniques and require fresh frozen tissues. By contrast, formalin-fixed paraffin-embedded (FFPE) tissue samples have been collected during decades of routine histopathological examination and those are the most widely available materials in clinical use. However, formaldehyde-containing fixatives cause cross-linkage between nucleic acids and proteins, rendering subsequent extraction and quantification of RNA challenging (1). A major obstacle to RNA expression analysis of FFPE tissues has been the uncertainty as to whether or not gene expression analyses from routinely archived tissues accurately reflect the expression levels prior to fixation, since the fixation process is likely to cause a high degree of RNA fragmentation (2). Given that naturally occurring small RNAs are not affected by fragmentation and therefore do not experience loss of quality, targeting miRNAs is more suitable for analysis of RNA extracted from FFPE samples.

miRNAs are 18- to 25-nucleotide, non-coding RNA molecules that regulate the translation of several genes (3). miRNA expression levels are altered in most types of human cancers (4-6). Several RCC studies have examined miRNAs by microarray analysis using a relatively small amount of frozen tissue samples. Various miRNAs that are dysregulated in RCC have been identified $(7,8)$. Several lines of evidence indicate that $m i R-486$ has oncogenic properties. Overexpression of miR-486 has been reported in cutaneous T-cell lymphoma (9). High serum or plasma expression levels of miR-486 have been reported in non-small cell lung (10) and gastric cancers (11). $m i R-486$ has also been reported to target PTEN and FOXO1, which negatively affect PI3K/Akt signaling (12). The Akt-signaling pathway functions as a potent activator of growth and survival signaling (13). However, expression of $m i R-486$ has not been investigated in RCC.

In the present study, we investigated the association of $m i R-486$ expression with RCC patient prognosis $(\mathrm{n}=150)$ using 
quantitative reverse transcription polymerase chain reaction (qRT-PCR) of FFPE samples. In addition, since it has been reported that olfactomedin 4 (OLFM4), which encodes the olfactomedin 4 protein, is one of the direct targets of $m i R-486$ (14), immunohistochemical analysis of olfactomedin 4 was carried out in RCC tissues.

\section{Materials and methods}

Tissue samples. In a retrospective study design, 150 primary tumors were collected consecutively from patients diagnosed with RCC who underwent surgery between 2001 and 2008 at the Hiroshima University Hospital (Hiroshima, Japan). Only patients without preoperative radiotherapy or chemotherapy were enrolled in the study. Of 150 patients, 129 were at stages I, II and III, while 21 were at stage IV. The 129 patients with stage I, II and III RCC underwent curative resection. Of the 129 patients with stage I, II and III RCC, 20 received adjuvant therapy (interferon- $\alpha$ ) (15). Of the 21 patients with stage IV RCC, 17 received postoperative therapy using interferon- $\alpha$ (15). Postoperative follow-up was scheduled every 1,2 or 3 months during the first 2 years after surgery, and every 6 months thereafter unless more frequent follow-up was deemed necessary. Chest-abdominal computed tomography scan and serum chemistries were performed during each 6 month visit at least. Patients were monitored by their physician until they succumbed to the disease or the date of the last documented contact. The median follow-up period was 64 months (range, 2-120). Operative mortality was defined as death within 30 days of the patient leaving the hospital, and these patients were omitted from the analysis.

For qRT-PCR analysis, archival FFPE tissues were used. Histological classification was based on the World Health Organization system. RCC cases were classified into clear cell RCC (ccRCC) and non-ccRCC. Tumor staging was performed according to the TNM grouping system. Since written informed consent was not obtained, for reasons of strict privacy protection, identifying information for the samples was removed prior to analysis. This procedure was in accordance with the Ethical Guidelines for Human Genome/Gene Research of the Japanese Government.

$R N A$ extraction and $q R T-P C R$. FFPE samples were sectioned $(10 \mu \mathrm{m})$, deparaffinized and stained with hematoxylin and eosin (H\&E) to ensure that the sectioned block contained tumor cells. The tumor areas in the adjacent sections were marked under a light microscope without hematoxylin staining. Tumor areas were macrodissected with sterile disposable scalpels and subjected to RNA isolation using the Recover All ${ }^{\mathrm{TM}}$ Total Nucleic Acid Isolation kit (Ambion, Austin, TX, USA), according to the manufacturer's instructions. Expression levels of $m i R-486$ and $R N U 6 B$ were measured using TaqMan ${ }^{\circledR}$ assays for miRNA (Applied Biosystems, Austin, TX, USA) in such a manner that the identity and clinical outcomes of samples were blinded. Complementary DNA (cDNA) was synthesized using miRNA-specific primers and the TaqMan ${ }^{\circledR}$ MicroRNA Reverse Transcription kit (Applied Biosystems) according to the manufacturer's instructions. Briefly, $40 \mathrm{ng}$ of RNA was reverse transcribed in a $20 \mu \mathrm{l}$ reaction with gene-specific RT probes. qRT-PCR was performed using the ABI 7900 Version
Table I. Association between miR-486 expression and clinicopathological characteristics.

\begin{tabular}{|c|c|c|c|}
\hline \multirow[b]{2}{*}{ Characteristics } & \multicolumn{3}{|c|}{ miR-486 expression } \\
\hline & High (\%) & Low & P-value \\
\hline \multicolumn{4}{|l|}{ Age (years) } \\
\hline$<66$ & $48(69)$ & 22 & 0.1084 \\
\hline$\geq 66$ & $64(80)$ & 16 & \\
\hline \multicolumn{4}{|l|}{ Gender } \\
\hline Male & $80(73)$ & 29 & 0.5552 \\
\hline Female & $32(78)$ & 9 & \\
\hline \multicolumn{4}{|l|}{$\mathrm{T}$ classification } \\
\hline 1 & $71(77)$ & 21 & 0.5009 \\
\hline 2 & $12(71)$ & 5 & \\
\hline 3 & $27(69)$ & 12 & \\
\hline 4 & $2(100)$ & 0 & \\
\hline \multicolumn{4}{|l|}{ N classification } \\
\hline 0 & $107(76)$ & 34 & 0.4049 \\
\hline 1 & $2(50)$ & 2 & \\
\hline 2 & $3(60)$ & 2 & \\
\hline \multicolumn{4}{|l|}{ M classification } \\
\hline 0 & $98(75)$ & 32 & 0.6119 \\
\hline 1 & $14(70)$ & 6 & \\
\hline \multicolumn{4}{|l|}{ Tumor stage } \\
\hline I & $71(79)$ & 19 & 0.1792 \\
\hline II & $10(71)$ & 4 & \\
\hline III & $16(67)$ & 8 & \\
\hline IV & $15(68)$ & 7 & \\
\hline \multicolumn{4}{|c|}{ Histological classification } \\
\hline $\mathrm{ccRCC}$ & $101(74)$ & 36 & 0.3649 \\
\hline Non-ccRCC & $11(85)$ & 2 & \\
\hline \multicolumn{4}{|l|}{ Venous invasion } \\
\hline Positive & $35(74)$ & 12 & 0.9699 \\
\hline Negative & $77(75)$ & 26 & \\
\hline \multicolumn{4}{|c|}{ Interferon- $\alpha$ treatment } \\
\hline Received & $23(62)$ & 14 & 0.0519 \\
\hline Not received & $89(79)$ & 24 & \\
\hline
\end{tabular}

ccRCC, clear cell renal cell carcinoma.

2.3 Sequence Detection System (Applied Biosystems). RNU6B was used as an endogenous normalization control for $m i R-486$. The assays were performed in triplicate. Quantification of miR-486 relative expression was calculated using the RQ manager 1.2 (Applied Biosystems).

Immunohistochemistry. FFPE samples were sectioned, deparaffinized and stained with $H \& E$ to ensure that the sectioned block contained tumor cells. Adjacent sections were then stained immunohistochemically with the Dako EnVision+ Mouse Peroxidase Detection System (DakoCytomation, Carpinteria, CA, USA). Antigen retrieval 
A

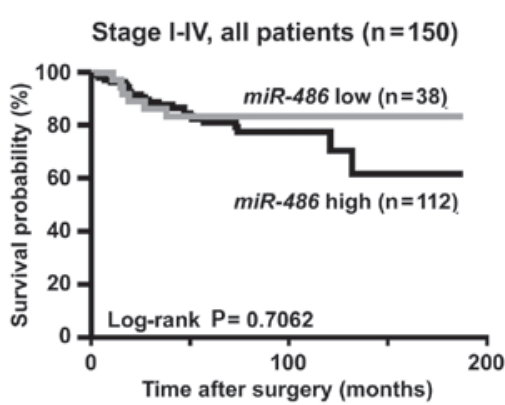

C

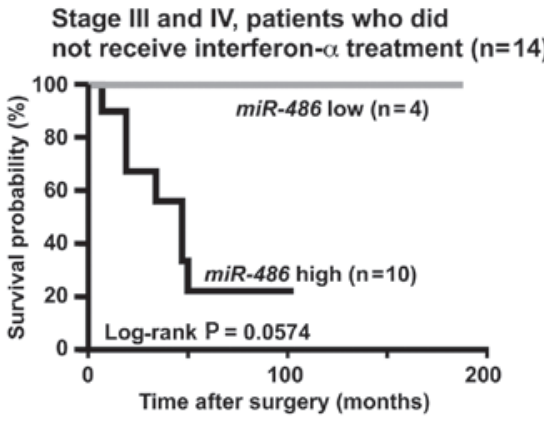

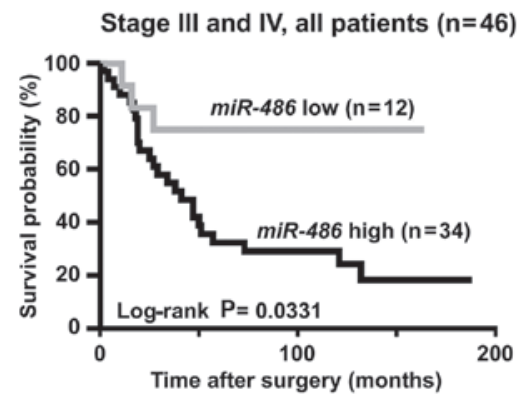

D

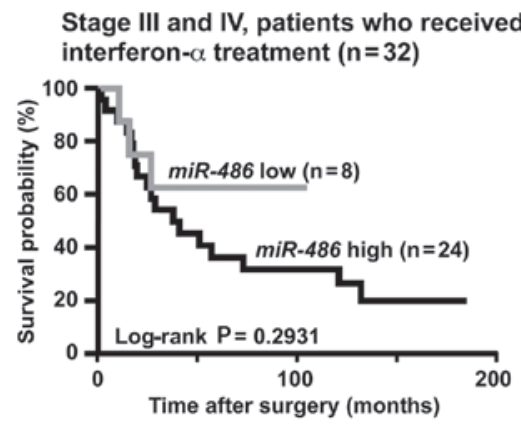

Figure 1. (A) Kaplan-Meier curves of miR-486-high or -low renal cell carcinoma (RCC) in stage I-IV patients. Cancer-specific mortality did not statistically vary in high- and low-miR-486 cases. (B) Kaplan-Meier curves of miR-486-high or -low RCC in stage III and IV patients. High-miR-486 expression had significantly worse cancer-specific mortality compared to those with low-miR-486 expression. (C) Kaplan-Meier curves of miR-486-high or -low RCC in stage III and IV patients who did not receive interferon- $\alpha$ treatment. Cancer-specific mortality of patients with high-miR-486 expression tended to be worse compared to that of patients with low-miR-486 expression. (D) Kaplan-Meier curves of miR-486-high or -low RCC in stage III and IV patients who received interferon- $\alpha$ treatment. Expression of miR-486 was not significantly associated with therapeutic outcome.

was performed by microwave heating in citrate buffer ( $\mathrm{pH} 6.0)$ for $30 \mathrm{~min}$. After peroxidase activity was blocked with $3 \%$ $\mathrm{H}_{2} \mathrm{O}_{2}$ methanol for 10 min, sections were incubated with normal goat serum (DakoCytomation) for 20 min to block non-specific antibody binding sites. Sections were incubated with primary antibodies against olfactomedin 4 (1:50 dilution, using an anti-olfactomedin 4 antibody raised in our laboratory) (16) for $1 \mathrm{~h}$ at room temperature, followed by incubations with EnVision+ anti-mouse peroxidase for $1 \mathrm{~h}$. Staining was completed with 10-min incubation with the substrate-chromogen solution. Sections were counterstained using $0.1 \%$ hematoxylin.

Statistical analysis. Associations between clinicopathological characteristics and $m i R-486$ expression were analyzed using the Fisher's exact test. To evaluate the associations between clinical covariates and cancer-specific mortality univariate and multivariate Cox regression analysis was used and conducted using SPSS software (SPSS Inc., Chicago, IL, USA). Hazard ratio (HR) and 95\% confidence interval (CI) were estimated from the Cox proportional hazard models. For the analyses, age was treated as a categorical variable ( 65 plus $>65$ vs. $<65$ years old). For the final multivariate Cox regression models, the variables that were moderately associated $(\mathrm{P}<0.10)$ with cancer-specific mortality were included. Differences in miR-486 expression levels between the two groups were determined by the Mann-Whitney U test using Graphpad Prism 5.0 (Graphpad Software, Inc., San Diego, CA, USA). Kaplan-Meier survival curves were constructed for high- and low-miR-486 patients using the Graphpad Prism 5.0 software. Differences between survival curves were tested for statistical significance using the log-rank test. $\mathrm{P}<0.05$ was considered to indicate a statistically significant difference.

\section{Results}

Expression of miR-486 in RCC and non-neoplastic kidney tissue. The expression of miR-486 and the corresponding non-neoplastic kidney tissue samples were assessed in $86 \mathrm{RCC}$ cases using qRT-PCR. The expression of $m i R-486$ in RCC samples was $\sim 2.7$-fold higher compared to that in corresponding non-neoplastic kidney samples $(\mathrm{P}<0.0001$, Mann-Whitney U-test).

Association between miR-486 expression levels and clinicopathological characteristics. Expression of miR-486 was examined in 64 additional RCC tissue samples using qRT-PCR. We investigated the association between the clinicopathological characteristics and miR-486 expression levels in 150 RCC cases (Table I). These 150 RCC cases were divided into high- and low-miR-486 cases. When low-miR-486 expression was classified according to the lowest quartile, the number of high- and low-miR-486 cases was 112 and 38, respectively. Expression of $m i R-486$ was not associated with clinicopathological characteristics such as gender, $\mathrm{T}, \mathrm{N}$ and $\mathrm{M}$ classifications, tumor stage or venous invasion.

Association between miR-486 expression and survival. The association between $m i R-486$ expression levels and cancer-specific mortality was evaluated. The Kaplan-Meier analysis for the 150 RCC cases (stages I-IV) was performed. 
Table II. Univariate and multivariate Cox regression analysis of miR-486 expression and cancer-specific mortality in stage III and IV RCC.

\begin{tabular}{|c|c|c|c|c|}
\hline \multirow[b]{2}{*}{ Characteristics } & \multicolumn{2}{|c|}{ Univariate analysis } & \multicolumn{2}{|c|}{ Multivariate analysis } \\
\hline & HR $(95 \%$ CI $)$ & P-value & $\mathrm{HR}(95 \% \mathrm{CI})$ & P-value \\
\hline \multicolumn{5}{|l|}{ Age (years) } \\
\hline$<66$ & 1 (Ref.) & 0.5719 & & \\
\hline$\geq 66$ & $1.24(0.58-2.66)$ & & & \\
\hline \multicolumn{5}{|l|}{ Gender } \\
\hline Male & 1 (Ref.) & 0.5919 & & \\
\hline Female & $0.73(0.17-2.08)$ & & & \\
\hline \multicolumn{5}{|c|}{ Histological classification } \\
\hline Non-ccRCC & 1 (Ref.) & 0.5714 & & \\
\hline $\mathrm{ccRCC}$ & $0.64(0.18-4.00)$ & & & \\
\hline \multicolumn{5}{|l|}{ Venous invasion } \\
\hline Negative & 1 (Ref.) & 0.9318 & & \\
\hline Positive & $1.04(0.42-3.10)$ & & & \\
\hline \multicolumn{5}{|l|}{$\mathrm{T}$ classification } \\
\hline $1 / 2$ & 1 (Ref.) & 0.8861 & & \\
\hline $3 / 4$ & $1.09(0.37-4.60)$ & & & \\
\hline \multicolumn{5}{|l|}{$\mathrm{N}$ classification } \\
\hline 0 & 1 (Ref.) & 0.0315 & 1 (Ref.) & 0.9832 \\
\hline $1 / 2$ & $2.73(1.10-6.24)$ & & $1.01(0.32-2.89)$ & \\
\hline \multicolumn{5}{|l|}{ M classification } \\
\hline 0 & 1 (Ref.) & 0.0035 & 1 (Ref.) & 0.2398 \\
\hline 1 & $3.16(1.46-7.08)$ & & $0.31(0.05-2.47)$ & \\
\hline \multicolumn{5}{|l|}{ Tumor stage } \\
\hline III & 1 (Ref.) & 0.0005 & 1 (Ref.) & 0.0386 \\
\hline IV & $4.03(1.82-9.54)$ & & $9.72(1.14-59.82)$ & \\
\hline \multicolumn{5}{|c|}{ Interferon- $\alpha$ treatment } \\
\hline Not received & 1 (Ref.) & 0.5558 & & \\
\hline Received & $1.28(0.57-3.28)$ & & & \\
\hline \multicolumn{5}{|c|}{$m i R-486$ expression } \\
\hline Low & 1 (Ref.) & 0.0202 & 1 (Ref.) & 0.0064 \\
\hline High & $3.38(1.18-14.26)$ & & $4.33(1.45-18.71)$ & \\
\hline
\end{tabular}

RCC, renal cell carcinoma; ccRCC, clear cell RCC; HR, hazard ratio; CI, confidence interval.

A

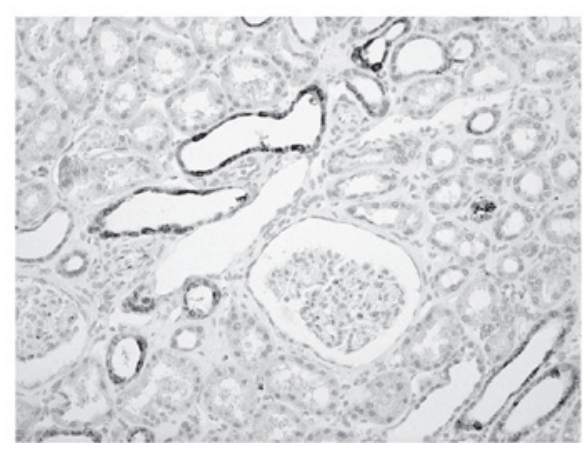

$\mathbf{B}$

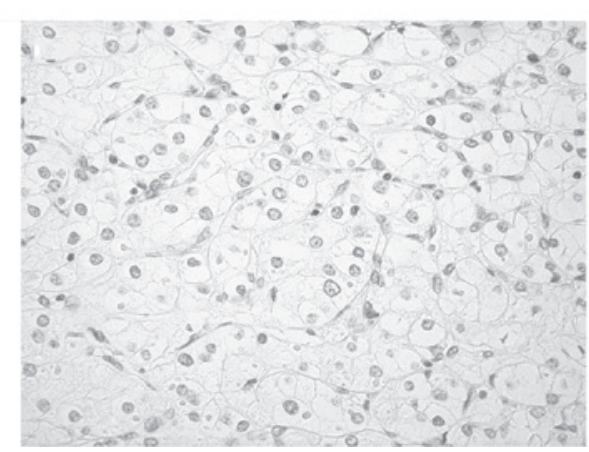

Figure 2. Immunohistochemical analysis of olfactomedin 4. (A) In non-neoplastic kidney, olfactomedin 4 staining was observed in uriniferous tubule but not glomerulus (original magnification, x200). (B) In renal cell carcinoma, olfactomedin 4 staining was not present (original magnification, x400). 
As shown in Fig. 1A, cancer-specific mortality did not statistically vary in high- and low-miR-486 cases.

Patients with RCC at stage I and II have a good rate of survival, whereas it is difficult to predict patient survival with stage III and IV RCC. These patients would benefit greatly from prognostic biomarkers. Therefore, we analyzed the prognostic value of $m i R-486$ expression in patients with stage III and IV RCC $(n=46)$. Using the Kaplan-Meier analysis, we found that RCC cases with high-miR-486 expression had significantly worse cancer-specific mortality compared to those with low-miR-486 expression $(\mathrm{P}=0.0331$, log-rank test, Fig. 1B). Since treatment with interferon- $\alpha$ for patients with stage III and IV RCC may affect patient survival, individuals who did not receive interferon- $\alpha$ treatment $(n=14)$ were assessed. Although cancer-specific mortality among patients with high- and low-miR-486 expression exhibited no statistically significant difference $(\mathrm{P}=0.0574, \log$-Rank test, Fig. $1 \mathrm{C})$, cancer-specific mortality of patients with high-miR-486 expression tended to be worse compared to that of patients with low-miR-486 expression.

To evaluate the potential for $m i R-486$ expression as a prognostic predictor in patients with stage III and IV RCC, univariate and multivariate Cox proportional hazards analyses were used to evaluate the association of $m i R-486$ expression with cancer-specific mortality (Table II). Findings of the univariate analysis showed that $\mathrm{N}$ (HR, 2.73; 95\% CI, 1.10-6.24; $\mathrm{P}=0.0315)$ and $\mathrm{M}$ classifications $(\mathrm{HR}, 3.16 ; 95 \% \mathrm{CI}$, 1.46-7.08; $\mathrm{P}=0.0035)$, tumor stage $(\mathrm{HR}, 4.03 ; 95 \% \mathrm{CI}$, 1.82-9.54; $\mathrm{P}=0.0005)$ and $m i R-486$ expression (HR, 3.38; $95 \% \mathrm{CI}, 1.18-14.26 ; \mathrm{P}=0.0202)$ were significantly associated with cancer-specific mortality. According to the multivariate model, which included $\mathrm{N}$ and $\mathrm{M}$ classifications, tumor stage and $m i R-486$ expression, expression of $m i R-486$ was an independent prognostic classifier of cancer-specific mortality (HR, 4.33; 95\% CI, 1.45-18.71; $\mathrm{P}=0.0064)$.

Survival analysis was performed using an alternatively defined cut-off point. When low-miR-486 expression was defined on the basis of lower than median levels of expression of $m i R-486$, the number of high- as well as low-miR-486 $m i R-486$ cases was 75 . Kaplan-Meier analysis revealed that cancer-specific mortality did not exhibit statistically significant differences in high- and low-miR-486 cases (data not shown). When low-miR-486 expression was classified according to the lowest tertile, the number of high- and low-miR-486 cases was 100 and 50, respectively. Kaplan-Meier analysis revealed that cancer-specific mortality did not exhibit statistically significant differences in high- and low-miR-486 cases (data not shown).

Expression of miR-486 and therapeutic outcomes. Biomarkers that predict therapeutic outcomes may provide tools to allow physicians to better stratify patients to more effective treatments. Therefore, we analyzed the association between $m i R-486$ expression and therapeutic outcomes in stage III and IV RCC patients treated with interferon- $\alpha(n=32)$. However, expression of $m i R-486$ was not significantly associated with therapeutic outcome ( $\mathrm{P}=0.2931$, log-rank test, Fig. 1D).

Expression of OLFM4 in RCC. OLFM4, which encodes the OLFM4 protein, has been reported as one of the direct targets of $m i R-486$ (14). Although alteration of OLFM4 expression has been reported in several types of human cancer (16-18), expression of OLFM4 in RCC has not been investigated. Therefore, the expression of OLFM4 was analyzed using immunohistochemistry in $86 \mathrm{RCC}$ cases. In non-neoplastic kidney, OLFM4 staining was observed in uriniferous tubules but not in glomeruli (Fig. 2A). By contrast, OLFM4 staining was not expressed in RCC cells (Fig. 2B). No OLFM4 staining was detectable in the 86 RCC cases.

\section{Discussion}

Several lines of evidence have suggested that miRNAs are useful as biomarkers as well as therapeutic targets for cancer. Prediction of the survival of patients with stage III and IV RCC remains difficult, and these groups would benefit from the detection of prognostic markers that identify individuals for whom adjuvant or post-operative treatment would be advantageous. In the present study, RNA from 150 FFPE RCC tissues was prepared, since biomarkers developed from FFPE samples may be more readily translated into clinical application. Expression of $m i R-486$ in patients with stage III and IV RCC was significantly associated with cancer-specific mortality according to a Kaplan-Meier analysis. The univariate and multivariate Cox proportional hazards analyses demonstrated that expression of $m i R-486$ was an independent prognostic classifier. Furthermore, since treatment with interferon- $\alpha$ for patients with stage III and IV RCC affects patient survival, individuals who did not receive interferon- $\alpha$ treatment were assessed. Although cancer-specific mortality among patients with high- and low-miR-486 expression showed no statistically significant difference, cancer-specific mortality of patients with high-miR-486 expression tended to be worse compared to that of patients with low-miR-486 expression. This finding suggests that $m i R-486$ expression is associated with a more aggressive RCC histology. Taken together, these results indicate that $m i R-486$ is a promising biomarker to identify patients with poor prognosis in stage III and IV RCC.

In the present study, we demonstrated that $m i R-486$ expression was associated with cancer-specific mortality in patients with stage III and stage IV RCC. In addition, $m i R$-486 expression was associated with cancer-specific mortality in stage III and IV RCC patients who were not treated with interferon- $\alpha$. Therefore, measurement of $m i R-486$ expression may help identify patients with a high risk of disease recurrence, while treatment with interferon- $\alpha$ may be indicative for patients with miR-486-positive RCC. However, it is unclear whether such patients may benefit from interferon- $\alpha$ treatment. To address this issue, we examined whether or not $m i R-486$ expression was able to identify patients for whom interferon- $\alpha$ treatment is beneficial in stage III and IV RCC. However, expression of $m i R-486$ was not significantly associated with therapeutic outcome. These results suggest that even when patients with a high risk of disease recurrence are identified by $m i R-486$ measurement, those patients may not benefit from interferon- $\alpha$ treatment. Novel therapeutic methods may be more effective for these patients.

$m i R-486$ has been reported to have oncogenic properties. Overexpression of $m i R-486$ has been reported in several human malignancies. In the present study, although expression of $m i R-486$ in RCC samples was significantly higher compared 
to that in corresponding non-neoplastic kidney samples, expression of $m i R-486$ was not associated with tumor stage. Therefore, high-miR-486 expression is likely to be involved in carcinogenesis, but not in tumor progression. Since targets of miR-486 are PTEN and FOXO1 (12), expression of PTEN and FOXO1 may be low in high-miR-486 RCC cases. In addition to PTEN and FOXO1, one of the targets of $m i R-486$ is OLFM4 (14). In the present study, although OLFM4 staining was observed in uriniferous tubules, staining of OLFM4 was not detected in the 86 RCC cases. Previously, we showed that patients with OLFM4-positive gastric cancer had a better survival rate compared to patients with OLFM4-negative gastric cancer (16). Forced expression of OLFM4 has also been reported to decrease cell adhesion and migration $(17,18)$. Taken together, OLFM4 is likely to have tumor suppressive properties in RCC. Given that staining of OLFM4 was not detected in the $86 \mathrm{RCC}$ cases, overexpression of $m i R-486$ is unlikely to have a major role in the loss of OLFM4.

In summary, we showed that a high-miR-486 expression is an independent prognostic classifier in stage III and IV RCC. Therefore, measurement of $m i R-486$ helps identify high-risk patients. In this study, expression of $m i R-486$ from FFPE samples was assessed. Therefore, measurement of $m i R-486$ can be readily translated into clinical applications.

\section{Acknowledgements}

This study was supported in part by grants-in-aid for Cancer Research from the Ministry of Education, Culture, Science, Sports and Technology of Japan and in part by a grant-in-aid for the Third Comprehensive 10-Year Strategy for Cancer Control from the Ministry of Health, Labor and Welfare of Japan. The authors would like to thank Mr. Shinichi Norimura for his excellent technical assistance and advice. This study was conducted in collaboration with the Research Center for Molecular Medicine of the Faculty of Medicine of Hiroshima University. We also thank the Analysis Center of Life Science of Hiroshima University for the use of their facilities.

\section{References}

1. Srinivasan M, Sedmak D and Jewell S: Effect of fixatives and tissue processing on the content and integrity of nucleic acids. Am J Pathol 161: 1961-1971, 2002.

2. Specht K, Richter T, Muller U, Walch A, Werner M and Hofler H: Quantitative gene expression analysis in microdissected archival formalin-fixed and paraffin-embedded tumor tissue. Am J Pathol 158: 419-429, 2001.
3. Huntzinger E and Izaurralde E: Gene silencing by microRNAs: contributions of translational repression and mRNA decay. Nat Rev Genet 12: 99-110, 2011.

4. Lu J, Getz G, Miska EA, et al: MicroRNA expression profiles classify human cancers. Nature 435: 834-838, 2005.

5. Schetter AJ, Heegaard NH and Harris CC: Inflammation and cancer: interweaving microRNA, free radical, cytokine and p53 pathways. Carcinogenesis 31: 37-49, 2010.

6. Ueda T, Volinia S, Okumura H, et al: Relation between microRNA expression and progression and prognosis of gastric cancer: a microRNA expression analysis. Lancet Oncol 11: 136-146, 2010.

7. Gottardo F, Liu CG, Ferracin M, et al: Micro-RNA profiling in kidney and bladder cancers. Urol Oncol 25: 387-392, 2007.

8. White NM, Bao TT, Grigull J, et al: miRNA profiling for clear cell renal cell carcinoma: biomarker discovery and identification of potential controls and consequences of miRNA dysregulation. J Urol 186: 1077-1083, 2011.

9. Narducci MG, Arcelli D, Picchio MC, et al: MicroRNA profiling reveals that miR-21, miR486 and miR-214 are upregulated and involved in cell survival in Sézary syndrome. Cell Death Dis 2: e151, 2011.

10. Hu Z, Chen X, Zhao Y, et al: Serum microRNA signatures identified in a genome-wide serum microRNA expression profiling predict survival of non-small-cell lung cancer. J Clin Oncol 28: 1721-1726, 2010.

11. Konishi H, Ichikawa D, Komatsu S, et al: Detection of gastric cancer-associated microRNAs on microRNA microarray comparing pre- and post-operative plasma. Br J Cancer 106: 740-747, 2012.

12. Small EM, O'Rourke JR, Moresi V, et al: Regulation of PI3-kinase/Akt signaling by muscle-enriched microRNA-486. Proc Natl Acad Sci USA 107: 4218-4223, 2010.

13. Crackower MA, Oudit GY, Kozieradzki I, et al: Regulation of myocardial contractility and cell size by distinct PI3K-PTEN signaling pathways. Cell 110: 737-749, 2002.

14. Oh HK, Tan AL, Das K, et al: Genomic loss of miR-486 regulates tumor progression and the OLFM4 antiapoptotic factor in gastric cancer. Clin Cancer Res 17: 2657-2667, 2011.

15. Medical Research Council Renal Cancer Collaborators: Interferon-alpha and survival in metastatic renal carcinoma: early results of a randomised controlled trial. Medical Research Council Renal Cancer Collaborators. Lancet 353: 14-17, 1999.

16. Oue N, Sentani K, Noguchi T, et al: Serum olfactomedin 4 (GW112, hGC-1) in combination with Reg IV is a highly sensitive biomarker for gastric cancer patients. Int J Cancer 125: 2383-2392, 2009.

17. Koshida S, Kobayashi D, Moriai R, Tsuji N and Watanabe N: Specific overexpression of OLFM4(GW112/HGC-1) mRNA in colon, breast and lung cancer tissues detected using quantitative analysis. Cancer Sci 98: 315-320, 2007.

18. Liu W, Liu Y, Zhu J, Wright E, Ding I and Rodgers GP: Reduced hGC-1 protein expression is associated with malignant progression of colon carcinoma. Clin Cancer Res 14: 1041-1049, 2008. 